\title{
Desarrollo de una aplicación web y móvil en tiempo real, una evolución de las aplicaciones actuales
}

Web And Mobile Application Development In Real Time, An Evolutionof Curr ent Applications

Ing. Loarte Cajamarca Byron Gustavo, MSc. ${ }^{1}$, Ing. Maldonado Soliz Ivonne Fernanda, M.Sc. ${ }^{2}$

Recibido: 12-10-2018 / Revisado: 27-11-2018 /Aceptado: 14-12-2018/ Publicado: 05-01-2019

\begin{abstract}
.
DOI: https://doi.org/10.33262/cienciadigital.v3i1.282

The necessary exponential evolution in the development of web and mobile applications produces new trends for companies to introduce and improve in them if they want to keep in the viable market. That is why applications are currently the instant response to any interaction, which in technology is known as Real time.

Real-time communication systems have become more important because it is imperative to be more and more connected to instant messaging applications, collaborative tools in graphics editing, and even online video games of virtual reality. The appearance of new tools for the development of these applications are the key to raise a general description on the operational of systems and applications in real time, rather than delve into each one of these aspects, which has been looking for is to offer the basic elements to have a wide vision of this kind of applications, together with the development of a web and mobile application with these new trends.
\end{abstract}

Keywords: Firebase, AngularJS, WebSocket, Real time, Ionic

\section{Resumen.}

El irrevocable crecimiento exponencial del desarrollo de aplicaciones web y móviles, provocan tendencia en que las empresas deban implementarlas si quieren seguir

\footnotetext{
1 Universidad Escuela Politécnica Nacional, Escuela de Formación de Tecnólogos, Quito, Ecuador, byron.loarteb@epn.edu.ec

2 Universidad Escuela Politécnica Nacional, Escuela de Formación de Tecnólogos, Quito, Ecuador, ivonne.maldonadof@epn.edu.ec
} 
siendo competitivos dentro del mercado. Es por ello que actualmente las aplicaciones son la respuesta inmediata a cualquier interacción, lo que en tecnología se conoce como Tiempo Real.

Los sistemas de comunicación en tiempo real han cobrado mayor importancia debido a que es imprescindible el estar cada vez más conectados ya sea a aplicaciones de mensajería instantánea, herramientas de colaboración en la edición de gráficos, y hasta videojuegos online en realidad virtual. El surgimiento de nuevas herramientas para el desarrollo de estas aplicaciones son la clave para plantear una descripción general sobre el funcionamiento de los sistemas y aplicaciones en tiempo real, más que profundizar en cada uno de estos aspectos, lo que se ha buscado es ofrecer los elementos básicos para tener una visión amplia de este tipo de aplicaciones, conjuntamente con el desarrollo de una aplicación web y móvil con estas nuevas tendencias.

Palabras claves: Tiempo Real, Firebase, AngularJS, WebSocket, Ionic

\section{Introducción}

En la actualidad es común ver en las PYMES diferentes tipos de aplicaciones ya sean de tipo: web, escritorio y móviles; todas y cada una de ellas cumpliendo roles primordiales por un lado mejorar la calidad de vida de las personas y por otra mejorar la gestión de la información en una organización.

Sin embargo, es por ello que en los últimos años diversas empresas han apostado por remodelar esta gama de aplicaciones para aprovecharse los beneficios que conlleva el uso y desarrollo de aplicaciones en tiempo real y con ello ofrecer una amplia gama de productos y servicios.

No obstante, aunque este termino de tiempo real, parecería que está muy en boga en la actualidad, no es para nada nuevo dentro de los conceptos de aplicaciones cliente/servidor que se ejecutan a través de internet, es por ello que es de vital importancia que cualquier persona que esté interesado en el desarrollo de estas aplicaciones conozcan los conceptos, funcionamiento, arquitectura y herramientas.

Antes de entender el concepto de aplicaciones en tiempo real y todo lo que conlleva es de vital importancia entender primeramente que son los Sistemas en tiempo real.

Un Sistema en tiempo real, no son más que Sistemas Informáticos que permiten una interacción dinámica entre los usuarios de dichos sistemas con el objetivo producir respuestas precisas dentro de unos límites de tiempo, esto supone que puedan enviarse respuestas inmediatas a las solicitudes realizadas entre los usuarios. 
En contra parte con cualquier Sistemas Informáticos frente a uno de tiempo real, supone que este último no solo va a depender del resultado lógico de la computación, sino que además depende del tiempo en el que el resultado se produjo, es por ello que una respuesta tardía es prácticamente una respuesta errónea". (Bennet, 1993)

De la misma manera cabe destacar que este tipo de Sistemas son utilizados en nuestra vida diaria por citar algunos, por ejemplo: Sistema de Radar, Control de tráfico aéreo, Servidores multimedia, Sistema de Satélite, Sistema de control y adquisición de datos, entre otros servicios que utilizamos diariamente.

Conociendo a breves rasgos lo que implica un Sistema en tiempo real, ahora se da paso para comprender que son las aplicaciones en tiempo real y del porque son indispensables en una era tecnológica de constantes cambios.

Una aplicación en tiempo real es un programa que nos permite establecer una comunicación bidireccional, dinámica y fluida, entre un servidor y los usuarios que tienen acceso a dicho servidor, es por ello que el servidor no solo se encarga en procesar los datos relativos a la información que va a dar a los usuarios finales, sino que además tiene que preocuparse de decidir con que usuario conectarse y cuándo hacerlo.

Un ejemplo claro en el cual se puede apreciar de mejor manera las principales ventajas que se tienen al usar aplicaciones en tiempo real son las siguientes:

- En este primer contexto es para las peticiones que se realizan a los servidores, imaginémonos que 100.000 clientes de un servicio deciden hacer una petición todos a la vez, aquí el servidor puede bloquearse por saturación de tantas peticiones. Sin embargo, ahora sí son los servidores quienes se van a encargar de gestionar las conexiones estas situaciones desaparecerían, ya que el servidor va a tener la capacidad de distribuir de forma normal todas las conexiones de forma que no haya problemas de saturación con las n peticiones de los clientes como se mencionó anteriormente.

- El siguiente ejemplo es algo obvio y es la instantaneidad de la información, es por ello que, si las aplicaciones tienen que preguntar al servidor normalmente lo hacen a intervalos. Los cuales se puede ir acumulando con el paso de tiempo y con ello obtener retardos bastante grandes. Ahora con aplicaciones en tiempo real, estos intervalos desaparecen y la información llega con un retraso mínimo.

- Finalmente, otra de las ventajas más considerables es la posibilidad de generar menos tráfico de red, que pasa si un cliente esta verificado cada cinco minutos si existen nuevos correos, cada cinco minutos va a estar generando tráfico si existen o no mensajes nuevos; ocasionando que se genere bastante tráfico inútil. Sin embargo, ahora si es el servidor es quien avisa al cliente de la existencia de un nuevo correo, ahora si es factible que se genere tráfico útil cuando existan nuevos correos. 
Como se puede apreciar en la actualidad la web tiende a ser un espacio interactivo en el cual ahora todas las comunicaciones cliente-servidor deben ser bidireccionales para que la información que se mueve sea en tiempo real. Es por ello que en este apartado se citaran aquellas herramientas que dispone los desarrolladores para atender estas nuevas necesidades.

Es importante mencionar que actualmente existen dos lados importantes para la construcción de estas aplicaciones en tiempo real: por un lado, el cliente (navegador web) y por otro lado el lado del servidor, el cual cuenta con una amplia gama de lenguajes de programación como: ASP.NET, PHP, Python, Ruby, Java etc. Mientras que del lado del cliente (navegador web) utiliza tecnologías como HTML, CSS y JavaScript para su estructura, diseño y programación.

De la misma manera como se mencionó anteriormente todas las aplicaciones en tiempo real son más bien aplicaciones bidireccionales, lo cual quiere decir que se puede enviar datos del cliente al servidor y de igual manera del servidor al cliente; todo esto es posible con la utilizando WebSocket.

Según (Garcia O., 2014) menciona "WebSocket prácticamente es mantener una conexión abierta entre un servidor web y un navegador, lo cual permite que tanto el navegador como el servidor envíen datos cuando lo deseen. Como la conexión es persistente, el intercambio de datos es muy rápido y se le se llama tiempo real."

Finalmente, con el constante crecimiento de las Tecnologías de la Información y con una amplia gama de herramientas, patrones, frameworks y metodologías para el desarrollo, ha permitido que las aplicaciones web se vuelvan cada vez más indispensables para la gestión de la información en las PYMES, permitiendo a los usuarios acceder a datos y servicios a través de internet. Para lo cual en el presente artículo se propone el desarrollo de una aplicación web en tiempo real abarcando todos los puntos citados anteriormente.

\section{Metodologia.}

Para el desarrollo de la aplicación web se utilizará Scrum, ya que es una metodología ágil donde la entrega de productos o servicios es primordial a fin de satisfacer los requisitos del cliente en pequeños incrementos iterativos que sean entregables tan pronto sea posible (Ramos D., 2017).

Sin embargo, esta metodología dispone de artefactos importantes como el product Backlog compuesto de una lista de tareas que llevan una descripción y estimación de esfuerzo y el sprint backlog el cual es un subconjunto de los elementos seleccionados en el product backlog.

Los artefactos mencionados anteriormente permitirán desarrollar el proyecto de manera organizada y en los tiempos establecidos. 
Cabe mencionar que cada uno de los requerimientos de la aplicación web serán recopilados en base a una serie de historias de usuario a fin de establecer la lista del product backlog. Cada uno de los requerimientos se ha distribuido en 3 iteraciones que se detallan a continuación.

\section{Primera Iteración}

En esta iteración se determina aquellas actividades que tiene que realizar los usuarios finales en la aplicación web, como se ilustra en la tabla 1.

Tabla 1: Primera iteración: Identificación de usuario en la aplicación web.

\begin{tabular}{lc}
\hline Enunciado de ítem de product backlog & Tiempo Estimado (horas) \\
\hline Identificación del usuario en la aplicación web & 20 horas \\
Registro de usuarios en la aplicación web & 40 horas \\
Visualizar usuarios en la aplicación web & 30 horas \\
Modificar usuarios en la aplicación web & 40 horas \\
Eliminar usuarios en la aplicación web & 40 horas \\
\hline
\end{tabular}

Elaborado por: Grupo de Investigadores.

\section{Segunda Iteración}

En esta iteración se determina aquellas actividades relacionadas para la gestión de productos en la aplicación web, como se ilustra en la tabla 2.

Tabla 2: Segunda iteración: Gestión de productos en la aplicación web.

\begin{tabular}{lc}
\hline \multicolumn{1}{c}{$\begin{array}{c}\text { Enunciado de ítem de product } \\
\text { backlog }\end{array}$} & $\begin{array}{c}\text { Tiempo Estimado } \\
\text { (horas) }\end{array}$ \\
\hline $\begin{array}{l}\text { Registro de productos en la aplicación } \\
\text { web }\end{array}$ & 40 horas \\
$\begin{array}{l}\text { Visualizar productos en la aplicación } \\
\text { web }\end{array}$ & 30 horas \\
Modificar productos en la aplicación web & 40 horas \\
Eliminar productos en la aplicación web & 40 horas \\
\hline
\end{tabular}

Elaborado por: Grupo de Investigadores.

\section{Tercera Iteración}

En esta iteración se determina aquellas actividades relacionadas para la visualización de productos en la aplicación móvil, como se ilustra en la tabla 3. 
Tabla 3: Segunda iteración: Visualización de productos en la aplicación móvil.

\begin{tabular}{lc}
\hline \multicolumn{1}{c}{ Enunciado de ítem de product backlog } & $\begin{array}{c}\text { Tiempo Estimado } \\
\text { (horas) }\end{array}$ \\
\hline $\begin{array}{l}\text { Identificación del usuario en la aplicación } \\
\text { móvil }\end{array}$ & 30 horas \\
$\begin{array}{l}\text { Visualización de productos en la aplicación } \\
\text { móvil }\end{array}$ & 30 horas \\
\hline
\end{tabular}

Elaborado por: Grupo de Investigadores.

\section{Resultados.}

Los resultados expresan como se fue procedió con el desarrollo de la aplicación web y móvil, de la misma manera se determina que herramientas se utilizaron, por ejemplo, para el desarrollo de la aplicación web se utilizó Angular JS el cual, permite desarrollar aplicaciones basadas en el patrón de arquitectura modelo vista controlador (MVC), cabe mencionar que su arquitectura se basa en JavaScript y HTML. Finalmente cabe recalcar que este Framework es completamente extensible y funciona adecuadamente con otras bibliotecas. Las características se pueden modificar o reemplazar para adaptarse a las necesidades del equipo de desarrollo (algo3.uqbar-project.org, 2018).

Mientras que para la aplicación móvil se utilizó el framework Ionic, el cual facilita el desarrollo de aplicaciones móviles hibridas basadas en HTML5, CSS3 y JS. No obstante, al tener una arquitectura central robusta, posibilita el desarrollo de aplicaciones de forma nativa para sistemas operativos con más demanda en el mercado que son: iOS y Android, finalmente cabe mencionar que este framework se encuentra optimizado con AngularJS (Ramos D., 2017).

Por ultimo para el almacenamiento de los datos y para que estos puedan ser accedidos en tiempo real, se utilizó la plataforma Firebase, el cual dentro de su amplia gama de servicio se utilizó Cloud Firestore, el cual permite mantener los datos sincronizados en tiempo real que se almacenan en documentos, organizados en colecciones y al ser un modelo de datos no relacional (NoSQL), admite cualquier estructura de datos (Firebase, 2018).

\section{Creación de la base de datos en la plataforma Firebase.}

En este apartado se procede a la creación de la base de datos, para lo cual se requiere como paso previo una cuenta de Google, la cual permite crear un proyecto en la proforma https://firebase.google.com/. Posterior a ello se procede con el inicio de sesión, se debe completar los siguientes campos como se ilustra en la figura 1.

- Seleccionar la opción para añadir proyecto 
- Ingresar el nombre del proyecto

- Seleccionar la región

- Aceptar los términos y condiciones

- Presionar el botón crear proyecto

Figura 1: Creación del proyecto en Firebase.

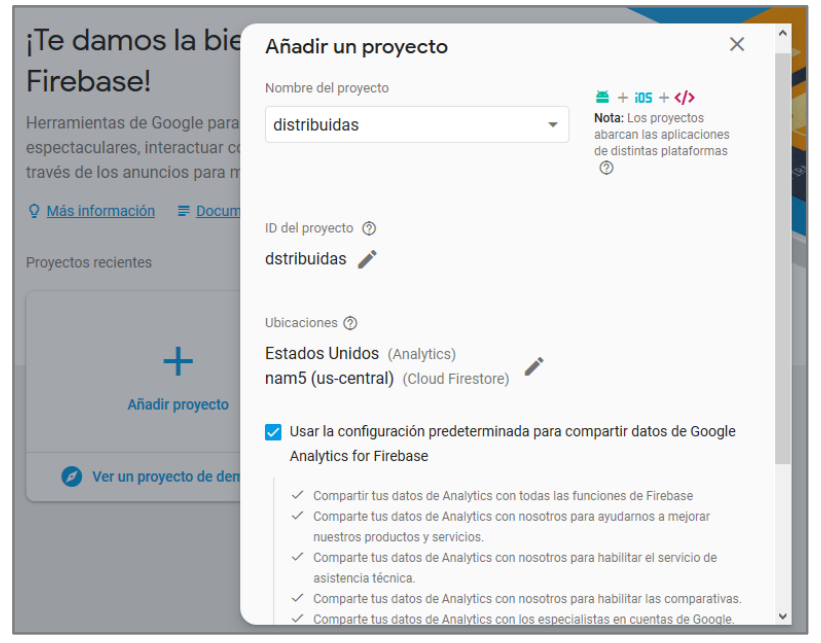

Elaborado por: Grupo de Investigadores.

Posterior e ello al seleccionar el tipo de almacenamiento que en este caso es Cloud Firestore, se procede a establecer los respectivos elementos y atributos que van a conformar la respectiva base de datos, creando las colecciones y documentos correspondientes como se puede ilustra en la figura 2.

Figura 2: Creación de colecciones en Cloud Firestore.

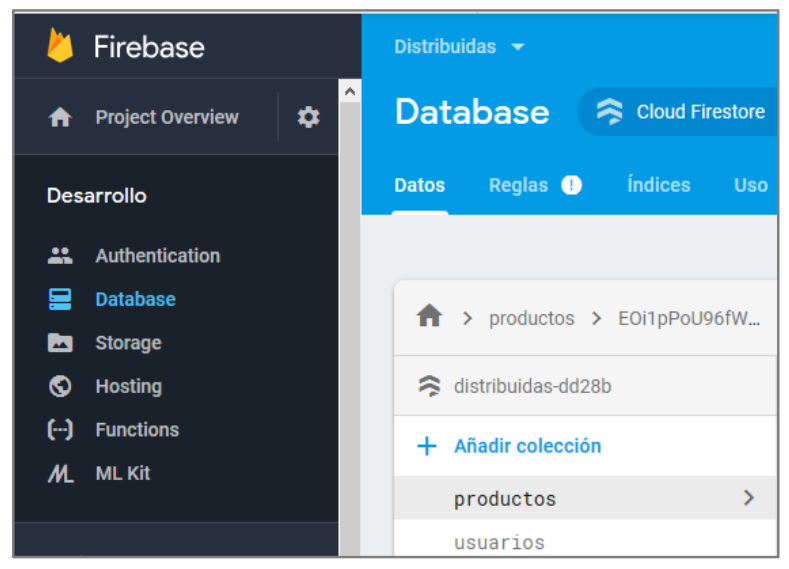

Elaborado por: Grupo de Investigadores. 
Una vez con la base de datos ya creada se procede con el desarrollo de la aplicación web, con el objetivo de cumplir cada uno de las iteraciones propuestas

\section{Identificación del usuario en la aplicación web}

Este módulo de la aplicación web será de uso exclusivo por el administrador, por ende, el usuario y las credenciales de acceso estarán definidos por el grupo de investigadores, como se ilustra en la figura 3.

Figura 3: Módulo de inicio de sesión del administrador.

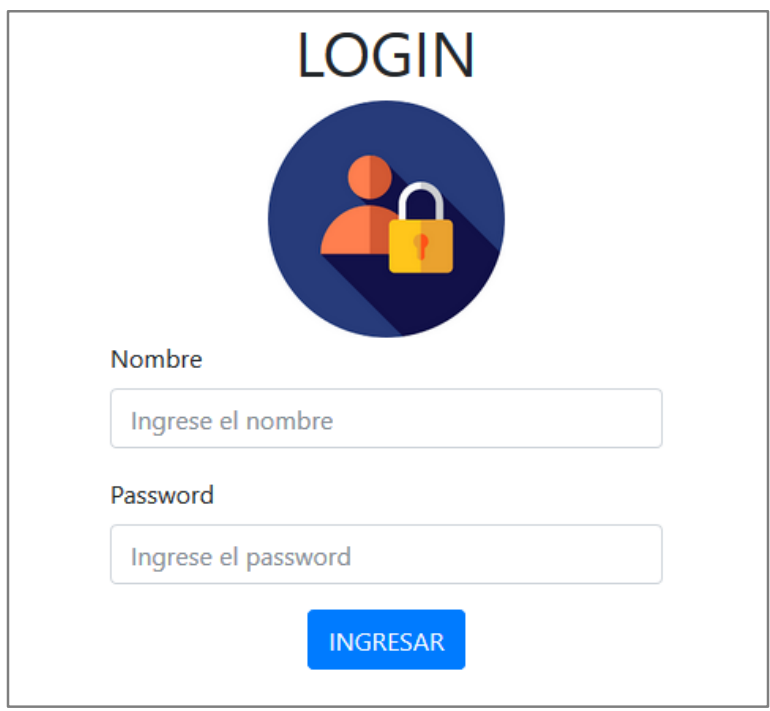

Elaborado por: Grupo de Investigadores.

\section{Registro de usuarios en la aplicación web}

Este módulo está conformado por un formulario de registro para registrar a nuevos usuarios en la aplicación web, como se ilustra en la figura 4.

Figura 4: Registro de usuarios.

Nombre
Nombre
Apellido
Apellido
Fecha de Nacimiento
dd $/ \mathrm{mm} /$ aaaa
Cédula
Cédula


Elaborado por: Grupo de Investigadores.

\section{Visualizar usuarios en la aplicación web}

En este módulo una vez que los usuarios ya están registrados en la base de datos se puede observar el listado de usuarios en la aplicación web, como se ilustra en la figura 5.

Figura 5: Visualización de usuarios.

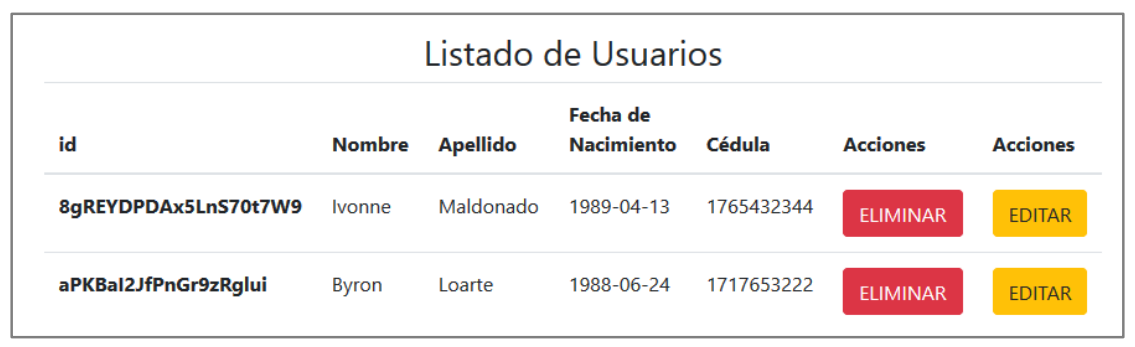

Elaborado por: Grupo de Investigadores.

\section{Modificar usuarios en la aplicación web}

Este módulo permite actualizar toda la información de un usuario que ya se encuentre previamente registrado en la aplicación web, de la misma manera al momento de actualizar la información el botón cambia de texto por actualizar datos, como se puede ilustra en la figura 6

Figura 6: Modificación de usuario.

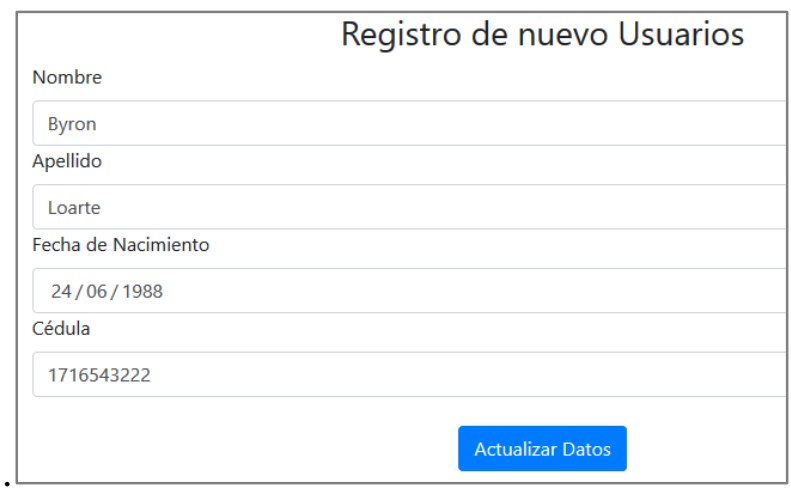

Elaborado por: Grupo de Investigadores.

\section{Eliminar usuarios en la aplicación web}

Finalmente, este módulo permite eliminar un usuario registrado en la aplicación web, datos que también serán eliminados automáticamente de la base de datos, como se ilustra en la figura 7 . 
Figura 7: Eliminación de usuario.

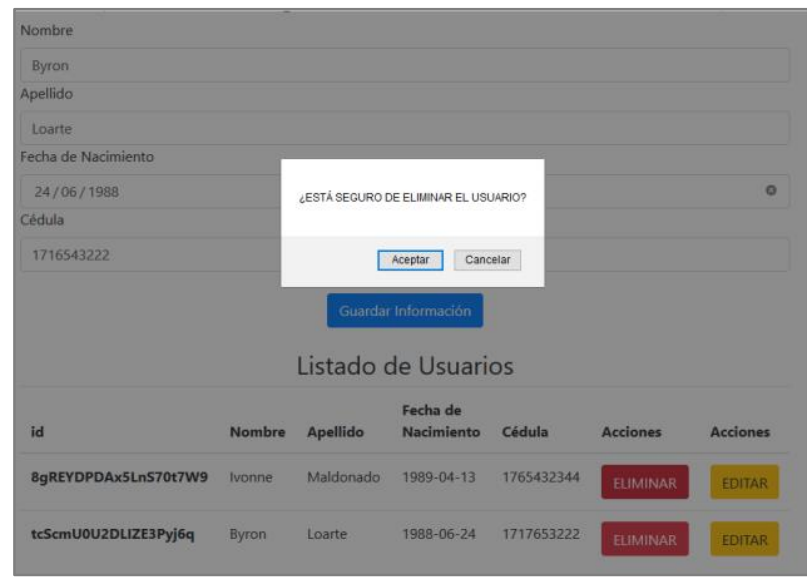

Elaborado por: Grupo de Investigadores.

A continuación, se procede con el desarrollo de siguiente iteración.

\section{Registro de productos en la aplicación web}

Este módulo está conformado por un formulario de registro, el cual permite registrar un nuevo producto en la aplicación web, como se ilustra en la figura 8.

Figura 8: Registro de productos.

\begin{tabular}{|l|}
\hline \multicolumn{1}{|c|}{ Registro de nuevos Productos } \\
Nombre \\
Nombre \\
Proveedor \\
Proveedor \\
Fecha de Ingreso \\
dd/mm/aaaa \\
Estado de Producto \\
Estado \\
\\
\end{tabular}

Elaborado por: Grupo de Investigadores.

\section{Visualizar productos en la aplicación web}

En este módulo una vez que los productos ya están registrados en la base de datos se puede observar el listado de nuevos productos en la aplicación web, como se ilustra en la figura 9. 
Figura 9: Visualización de productos.

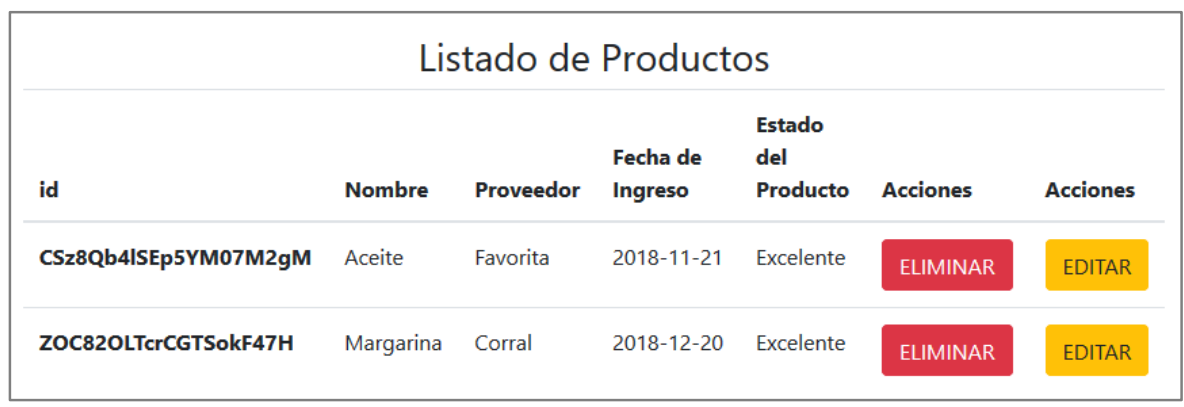

Elaborado por: Grupo de Investigadores.

\section{Modificar productos en la aplicación web}

Este módulo permite actualizar toda la información de un producto que ya se encuentre previamente registrado en la aplicación web, de la misma manera al momento de actualizar la información el botón cambia de texto por actualizar datos, como se puede ilustra en la figura 10 .

Figura 10: Modificación de producto.

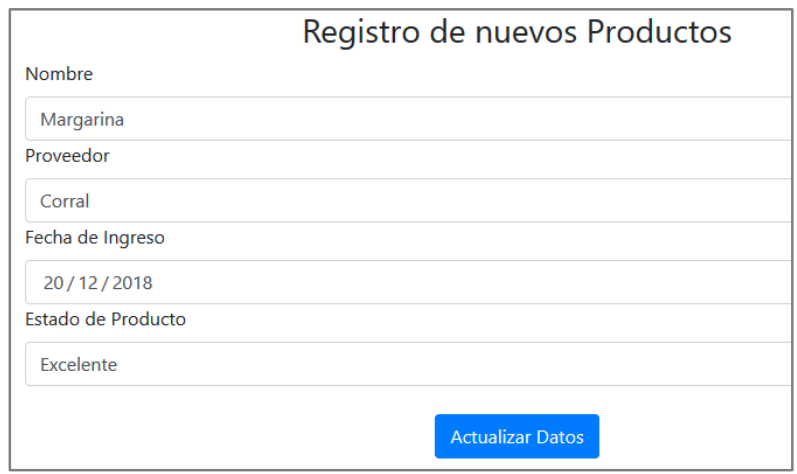

Elaborado por: Grupo de Investigadores.

\section{Eliminar productos en la aplicación web}

Finalmente, este módulo permite eliminar un producto registrado en la aplicación web, datos que también serán eliminados automáticamente de la base de datos, como se ilustra en la figura 11.

Figura 11: Eliminación de productos. 


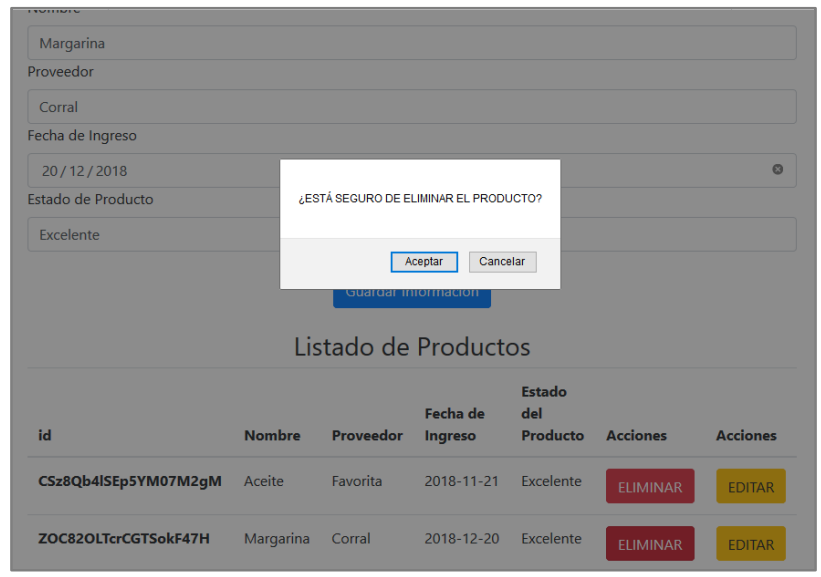

Elaborado por: Grupo de Investigadores.

Finalmente, para dar por concluido el desarrollo se procede a culminar las actividades de la tercera iteración.

\section{Identificación del usuario en la aplicación móvil}

Todos los usuarios que fueron registrados en la aplicación web pueden iniciar sesión automáticamente en la aplicación móvil para posterior a ello visualizar los productos registrados, como se ilustra en la figura 12.

Figura 12: Inicio de sesión del usuario.

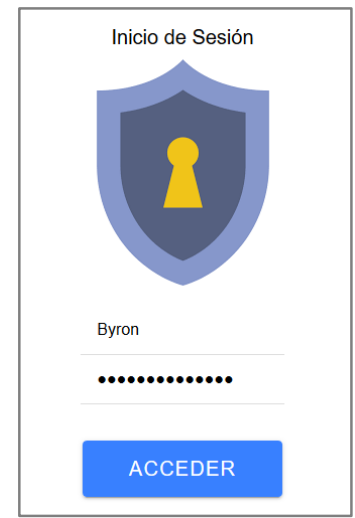

Elaborado por: Grupo de Investigadores.

\section{Visualización de productos en la aplicación móvil}

Este módulo de la aplicación móvil permite visualizar los respectivos productos que están registrados desde la aplicación web, de la misma manera se puede apreciar que por cada producto se puede insertar una imagen respectiva, como se ilustra en la figura 13.

Figura 13: Visualización de productos. 


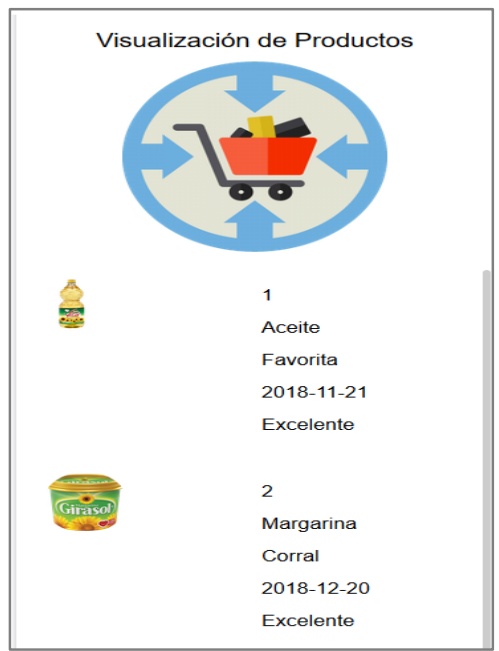

Elaborado por: Grupo de Investigadores.

\section{Pruebas de Aceptación}

Finalmente, para comprobar que toda la funcionalidad tanto de la aplicación web como de la aplicación cumplen se realizaron las perspectivas pruebas de aceptación. Es por ello que continuación se presentan las pruebas de aceptación más relevantes como se puede observar en las siguientes tablas.

Tabla 4: Prueba de aceptación: gestión de usuarios en la aplicación web.

\begin{tabular}{|l|l|}
\hline \multicolumn{2}{|l|}{ Prueba de aceptación } \\
\hline Identificador (ID): 1 & Identificador historia de usuario: $2,3,4$ y 5 \\
\hline Nombre prueba de aceptación: Gestión de usuarios en la aplicación web \\
\hline Descripción: \\
El usuario administrador tendrá la posibilidad de registrar, actualizar y eliminar \\
\hline Pasos de ejecución: \\
Ingresar datos del usuario. \\
Presionar el botón Guardar Información. \\
\hline Resultado deseado:
\end{tabular}


Al ingresar toda la información de los usuarios se debe visualizar toda la información en la aplicación web.

\section{Evaluación de la prueba:}

Se visualizó el resultado deseado.

Elaborado por: Grupo de Investigadores.

Tabla 4: Prueba de aceptación: gestión de productos en la aplicación web.

\begin{tabular}{|l|l|}
\hline \multicolumn{2}{|l|}{ Prueba de aceptación } \\
\hline Identificador (ID): 2 & $\begin{array}{l}\text { Identificador historia de usuario: } 9,7,8, \\
\text { Nombre prueba de aceptación: Gestión de productos en la aplicación web }\end{array}$ \\
\hline Descripción: \\
El usuario administrador tendrá la posibilidad de registrar, actualizar y eliminar \\
productos \\
\hline Pasos de ejecución: \\
Ingresar datos del producto. \\
Presionar el botón Guardar Información. \\
\hline Resultado deseado: \\
Al ingresar toda la información de los productos se debe visualizar toda la \\
información en la aplicación web. \\
\hline Evaluación de la prueba: \\
Se visualizó el resultado deseado.
\end{tabular}

Elaborado por: Grupo de Investigadores.

Tabla 4: Prueba de aceptación: visualización de productos en la aplicación móvil.

\section{Prueba de aceptación}




\section{\begin{tabular}{|l|l|}
\hline Identificador (ID): 1 & Identificador historia de usuario: 10 y 11
\end{tabular}}

Nombre prueba de aceptación: Visualización de productos en la aplicación móvil

\section{Descripción:}

El usuario que acceda a la aplicación móvil tendrá la posibilidad de visualizar todos los productos que están registrados en la aplicación web.

\section{Pasos de ejecución:}

Visualizar los datos de los productos.

\section{Resultado deseado:}

Al ingresar a la aplicación móvil toda la información de los productos se deben visualizar.

\section{Evaluación de la prueba:}

Se visualizó el resultado deseado.

Elaborado por: Grupo de Investigadores.

\section{Conclusiones.}

- La incorporación adecuada de herramientas permitió que mientras se iba desarrollando de la aplicación web y móvil se pueda evidenciar de mejor manera el funcionamiento de los datos en tiempo real, y como la información se fue obteniendo de manera instantánea sin necesidad de refrescar el navegador web.

- Actualmente la plataforma Firebase cuenta con una amplia gama de servicios. La incorporación de Cloud Firestore, permitió tener una base de datos de datos en tiempo real manteniendo de esta manera los datos sincronizados entre apps cliente a través de agentes de escucha en tiempo real.

- Firebase permitió guardar y sincronizar datos en la nube en tiempo real y a su vez ofreció la plataforma de administración unificada para gestionar la aplicación de el mismo proyecto, sin salir de este de esta API.

- Para el desarrollo de la aplicación web y móvil se elaboró con metodología ágil SCRUM, la cual permite crear ciclos cortos de iteraciones o sprints para poder alcanzar los objetivos planteados en el desarrollo del proyecto y acortar el periodo de entrega. 
- El desarrollo aplicaciones web y móviles han evolucionado en muchos sentidos en los últimos 10 años, y con ello las facilidades para programar diversas aplicaciones complejas han aumentado considerablemente. Es por eso que está en la mano de los programadores crear aplicaciones que expriman al máximo estas capacidades y que los principales beneficiados del manejo de información en tiempo real sean las empresas a futuro.

- Hoy en día el desarrollo de aplicaciones web y móviles ha creciendo de manera exponencial, debido a que facilitan a las personas estar en contacto y consultar información en el momento que lo desean, superando la barrera de distancia y convirtiéndose en la principal herramienta para estar conectados.

\section{Referencias bibliográficas.}

algo3.uqbar-project.org. (08 de 04 de 2018). AngularJS. Recuperado desde: http://algo3.uqbarproject.org/material/herramientas/angular/angular---conceptos-principales

Burns A., Wellings A. (2005). Sistemas de tiempo Real y Lenguajhes de Programación. Addison Wesley.

Crespo A., Alonso A. (Septiembre de 2010). Una Panorámica de los Sistemas de Tiempo Real. Recuperado desde: https://polipapers.upv.es/index.php/RIAI/article/view/8121

De la Puente J. (2001). Diseño de Sistemas de tiempo real. Recuperado desde: http://isa.uniovi.es/docencia/TiempoReal/Recursos/Transparencias/Desarrollo.pdf

Firebase. (29 de Mayo de 2018). Modelo de datos de Cloud Firestore. Recuperado desde: https://firebase.google.com/docs/firestore/data-model?hl=es-419

Garcia O. (Marzo de 2014). Aplicaciones en Tiempo Real con node.js. Recuperado desde: https://docplayer.es/5960427-Aplicaciones-en-tiempo-real-con-node-js-por-oscar-graciaaplicaciones-en-tiempo-real-con-node-js-marzo-2014-www-northware-mx.html

Graña Domínguez S. (Noviembre de 1997). Aportación al Diseño de Aplicaciones de Tiempo Real sobre Arquitecturas Paralelas. Recuperado desde: http://oa.upm.es/9813/1/SANTIAGO_GRANA_DOMINGUEZ.pdf

Ramos D., Noriega R., Laínez R., Durango A. (2017). Curso de Ingeniería de Software: $2^{\text {a }}$ Edición. CreateSpace Independent Publishing Platform.

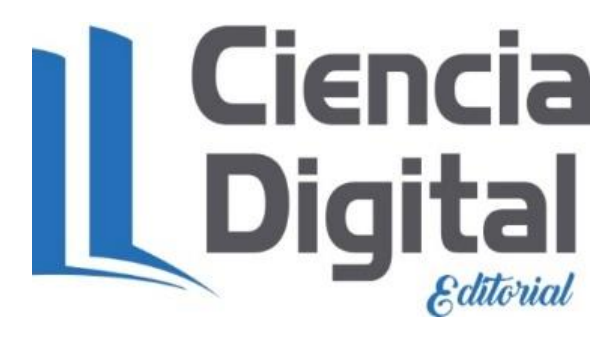


Para citar el artículo indexado.

Loarte B.\&, Maldonado I. (2019). Desarrollo de una aplicación web y móvil en tiempo real, una evolución de las aplicaciones actuales. Revista electrónica Ciencia Digital 3(1), 201-216. Recuperado desde:

http://cienciadigital.org/revistacienciadigital2/index.php/CienciaDigital/article/view/282/67 $\underline{9}$

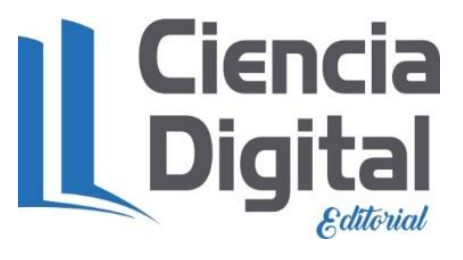

El artículo que se publica es de exclusiva responsabilidad de los autores y no necesariamente reflejan el pensamiento de la Revista Ciencia Digital.

El artículo queda en propiedad de la revista y, por tanto, su publicación parcial y/o total en otro medio tiene que ser autorizado por el director de la Revista Ciencia Digital.
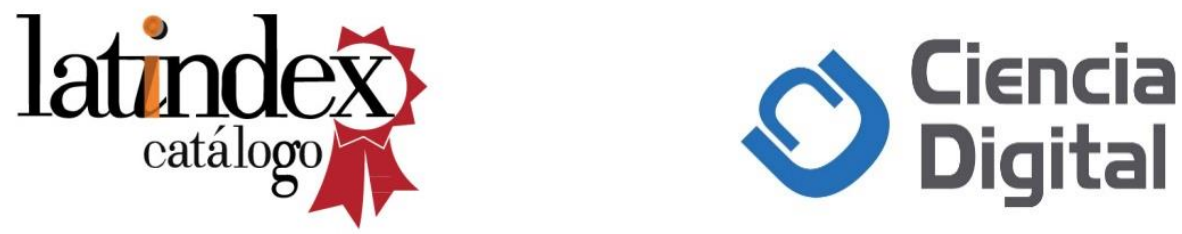